\title{
Ubiquitous Learning on Pocket SCORM
}

\author{
Hsuan-Pu Chang ${ }^{1}$, Wen-Chih Chang ${ }^{1}$, Yun-Long Sie ${ }^{1}$, Nigel H. Lin ${ }^{1}$, \\ Chun-Hong Huang ${ }^{1}$, Timothy K. Shih ${ }^{1}$, and Qun Jin ${ }^{2}$ \\ ${ }^{1}$ Department of Computer Science and Information Engineering, \\ Tamkang University, Taiwan, Republic of China \\ sul@mail.mine. tku. edu. tw \\ ${ }^{2}$ Department of Human Informatics and Cognitive Sciences, \\ Waseda University, Tokorozawa-shi, Saitama 359-1192, Japan \\ jin@waseda.jp
}

\begin{abstract}
With advanced technologies, computer devices have become smaller and powerful. As a result, many people enjoy ubiquitous learning using mobile devices such as Pocket PCs. Pocket PCs are easy to carry and use as a distance learning platform. In this paper, we focus on the issues of transferring the current PC based Sharable Content Object Reference Model (SCORM) to Pocket PC based. We will also introduce the Pocket SCORM Run-Time Environment (RTE) which has been developed in our lab. Pocket SCORM architecture is able to operate, even when the mobile device is off-line. It will keep the students' learning record. When it is on line, the records will then be sent back to Learning Management System (LMS). With memory limitation, we provides course caching algorithm to exchange the course content on the Pocket SCORM.
\end{abstract}

Keywords: Pocket PC, PDA, SCORM, Distance Education, Ubiquitous Learning.

\section{Introduction}

Because of the advantage in size, Pocket PC is easy to be carried on hand or in the pocket. This advantage makes it a suitable platform for distance education because ELearners can keep studying the course materials while they are away from their desk. Without the limitations of time and space, E-Learners can read their learning materials while they are walking, taking bus, and whenever they have leisure time to turn on their Pocket PCs. This new learning style provides some extra learning time for E-Learners who live in this rushing world.

Although Pocket PC devices have been improved in both computing power and memory storage, they are still with lots of limitation compared with laptop or desktop computers. The following points are some differences between the Pocket PC devices and laptop or desktop computers.

\section{Connection Mechanism}

Normally, E-Learners will be on-line when they are using laptop or desktop computers. Since E-Learners would stay at the same location longer when using their 
laptop or desktop computers than using a Pocket PC devices. As a result, they will be kept connected with the LMS, which is running on the server, all the time. This is different from Pocket PC devices because these devices are designed to be portable.

\section{Courseware Import and Export}

As we mentioned previously, Pocket PC devices sometimes could be disconnected from the network; however, we hope E-Learners are able to learn even when the network is not existed. In order to enable this functionality, we need to seek a way to allow the courseware to be temporary stored on the hand carried devices.

\section{Learning Records Buffering}

For a distance education standard such as SCORM, it requires the LMS to be able to keep tracking on learners' learning records so these records could be used to determine learners' progress or maybe transfer to other LMS where learner continue his education. As a result, it is important for a SCORM compatible platform to be able to store learners' learning records.

In this paper, we proposed a platform which focuses on the pocket devices. There is also a LMS which will be SCORM compliant to support the Pocket SCORM platform and be the major data store. The paper organization is listed as following. In Section 2, we introduce some related works of SCORM and systems on the pocket device. Section 3 shows the architecture of our Pocket SCORM architecture. In Section 4, we introduce the Pocket SCORM RTE which has been developed in our lab. In Section 5, we introduce the course caching algorithm in Pocket SCORM. Finally the conclusion and the future works are discussed in Section 6.

\section{Related Works}

Distance education enables E-Learners to learn without the restrictions of both time and space. There are many web-based courseware have been developed to allow learners to browse course content via a browser. SCORM (Sharable Content Object Reference Model) is a standard which is proposed by ADL (Advanced Distributed Learning) [1]. SCORM is aiming at the standardization of computer based teaching components. There are some papers related to SCORM have been published. Some advantages such as portability of learning content, the standardized communication interface between the LMS and WBTs, and supporting the reusability of learning object came alone with this new proposed standard. However, there are some problems as well such as the market value of SCOs, the process of producing WBTs on the basis of different SCO providers, the maintenance of SCOs and WBTs, and the quality of WBTs based on SCOs of different providers. A review discussed these issues can be found in [2]. Another paper demonstrated the Implementation of Content Repository Management System is referenced in [3]. In "Using SOAP and .NET web service to build SCORM RTE and LMS" [4], the XML Web Service based LMS and RTE was introduced. There was another system developed for automating the generation of SCORM-Based multimedia product training manuals was introduced in [5].

Personal Digital Assistants (PDAs) have become new learning tools for distance education in recent years. Portability of the PDAs was welcomed by students, and 
advantageous was advantageous, limitations such as the small screen size, navigation difficulties, and slow and error-prone methods for entering text, made it difficult to read and interact with document on the PDA [6]. Some students' experiences for reading course materials by PDAs were experimented. There are also some applications developed for educational purposes. TekPAC (Technical Electronic Knowledge Personal Assistant Capsule) was introduced in [7]. TekPAC was developed for providing access to readily available electronic information, allowing the user to perform tasks at locations with all schematics, photos, videos and BKMs readily available, and integrating key interventions to raise performance of target audience. PDAs have also been adopted in medical field as a tool for education. There were some PDA Projects at Virginia Commonwealth University being introduced in [8].

\section{Pocket SCORM Architecture}

In proposed architecture, we pointed out two types of connection for a Pocket PC to connect with LMS Server. One type is Pocket PC is connected to the server through wired or wireless network to the internet. The other is Pocket PC connects to the server via $\mathrm{PC}$ to the internet while Pocket $\mathrm{PC}$ is synchronizing with the PC.

In order to make courseware reusable, a standard representation of contents and structures must be enforced. The Content Aggregation Model (CAM) serves this purpose. CAM can be discussed in three parts: the Content Model, the Metadata, and the Content Packaging.

There are three major modules within the Pocket SCORM Architecture. In the following three sub-sections, we will show the details of each of them.

\subsection{Pocket SCORM Run-Time Environment}

There are six major components which are included in Pocket SCORM Run-Time Environment. All these components work together to form the whole Pocket SCORM Run-Time Environment. They are listed as below:

\section{Communication Agent}

The Communication Agent is used when the pocket devices try to communicate with the SCORM LMS Server. When E-Learners try to download the SCORM based courseware from the LMS Server, it will receive the packed courseware and pass it to Data UnPacking Agent. If there are some learning records need to be sent back to the LMS server, the Communication Agent will connect to the LMS Server and send the packed learning records back to the server. We considered using Simple Object Access Protocol (SOAP) [9] to be our transmission protocol to make our services of server side more extendable.

\section{Data Packing Agent}

We implemented the Data Packing Agent to reduce network load. This agent will pack the data before sending it to Communication Agent.

\section{Data UnPacking Agent}

Package Interchange File (PIF) is the exchange file format. Therefore, we need Data UnPacking Agent to unpack the PIF file. 


\section{Learning Agent}

The Learning Agent will keep tracking on the learner's learning records when the course is started. The learner might be off-line, the Learning Agent stores those learning records in the SCORM PDA Database temporarily. After it is on-line, those temporary stored learning records will be sent back to the Server.

\section{SCORM PDA Reader}

Due to the small screen restriction, normal web-based course content is not suitable for learners. They might need to use a stylus to control the scrollbars inside the Pocket Browser. It is inconvenient for the learners to use. To overcome this drawback, we designed "Reflow" function in the SCORM PDA Reader. "Reflow" function will adjust the content to make it fit in the width of the display width on our reader.

\section{SCORM PDA Database}

SCORM PDA Database has the capability of storing the temporary learners' learning records and the downloaded courseware.

\subsection{PC Dock}

If the Pocket PC without the ability to be on-line, it will require a PC Dock to be able to connect to the LMS server. There is a Synchronization Agent inside the PC Dock. The Synchronization Agent will perform the data transmission job between Communication Agent on the Pocket SCORM RTE and XML Web Service on the SCORM LMS Server.

\subsection{SCORM LMS Server}

There are two major components involved in SCORM LMS Server. One is the SCORM Data Repository, and the other is Pocket SCORM Service API. SCORM LMS Server provides distance education courseware which follows SCORM Data Model definition. The learners' information is also saved in the SCORM LMS Server. When a learner connects to the LMS Server, he or she needs to first logon the system before he or she can access any course materials. The usage of these two major components is stated as below:

\section{SCORM Data Repository}

The SCORM Data Repository stores all the course materials which follow SCORM Data Object Model. In the paper, we mainly focused on the Pocket PC devices. Therefore, we only care about how SCORM Data Repository interacts with our Pocket SCORM Service API. Nevertheless, this data repository should also support any SCORM based API. Furthermore, learners' learning records are also stored in this data repository. These SCORM based learning records should also be able to interact by using either Pocket SCORM Service API or any SCORM based API.

\section{Pocket SCORM Service API}

Ideally, Pocket SCORM Service APIs should be same as normal SCORM based APIs. We hope defined Pocket SCORM Service APIs can be widely applied by other applications. We tried to build Pocket SCORM Server APIs by adopting XML Web 
Service [10] technology. XML Web Service takes SOAP as its transmission protocol. One of the advantages of using XML Web Service as our APIs is the accessibility. Since SOAP is loosely coupled protocol by using XML wrapped envelope to invoke APIs, this vantage makes XML Service APIs can be accessed by any platform which follows SOAP protocol to acquire the service. As a result, we hope to implement our Pocket SCORM Service APIs as XML Web Services.

\section{Pocket SCORM RTE Implementation}

Based on the proposed architecture, we have been working on the implementation of the whole architecture. Up to present, we have completed some portion of the whole Pocket SCORM Architecture. There were some components which comprises the Pocket SCORM Run-Time Environment. Some user interfaces and functions will be introduced in this section.

In fig. 1, there are two user interfaces. On the left hand side, it shows the UI when student try to logon to Pocket SCORM RTE. Because we need to track on learners' learning records, the learner needs to provide his identity before studying the course materials. There is also an important issue need to be taken care. We need to make sure is the same user who is studying the courseware. In order to make sure learner's identity, the logon UI will be pop-up each time when Pocket PC has been turned off and turned back on again. On the right hand side of fig. 1, it shows a list of imsmanifest files which represent the each different course structure.

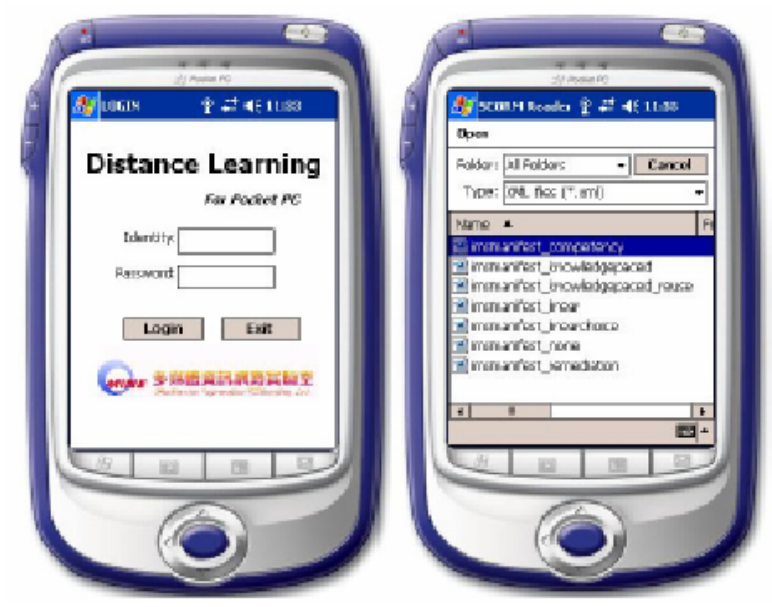

Fig. 1. Logon Interface and Course List View

In SCORM there are two major structures of a course were defined. One of them is knowledge based course structure, which is shown as on the left hand side of fig. 2, and the other one is linear based structure, which is shown as on the right hand side of fig. 2. Our Pocket SCORM RTE is capable to display the course structure according to defined imsmanifest file which learner chose to load. 

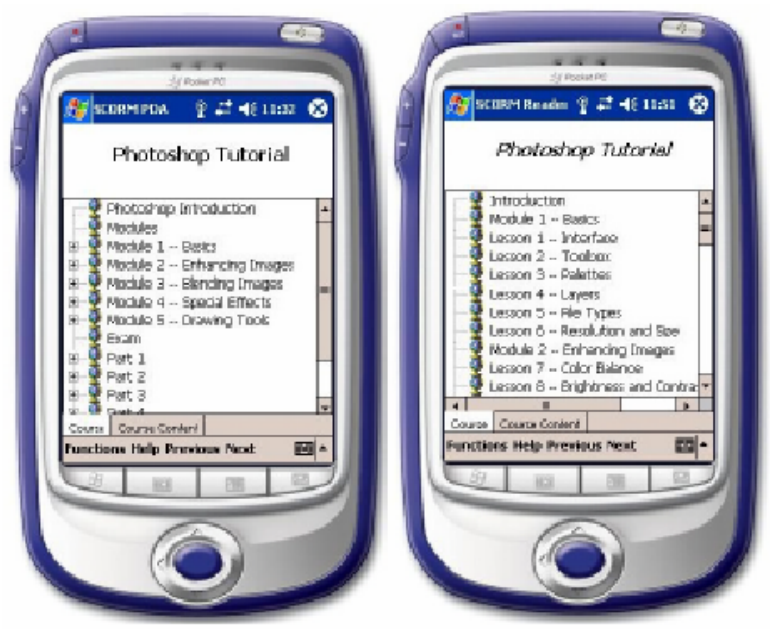

Fig. 2. Knowledge based and Linear based Structure

Fig. 3 has shown our SCORM PDA Reader and a glance of our SCORM PDA Database. On the left hand side of fig. 3, it shows the UI of SCORM PDA Reader. As we introduced in section 3, our SCORM PDA Reader provides "Reflow" function to enhance the ease of reading with one hand only. In order to gain better performance, we adopted SQL CE which is a compact version of Microsoft SQL Server. On the right hand side of fig. 3 , we have shown the database content within SQL CE database.
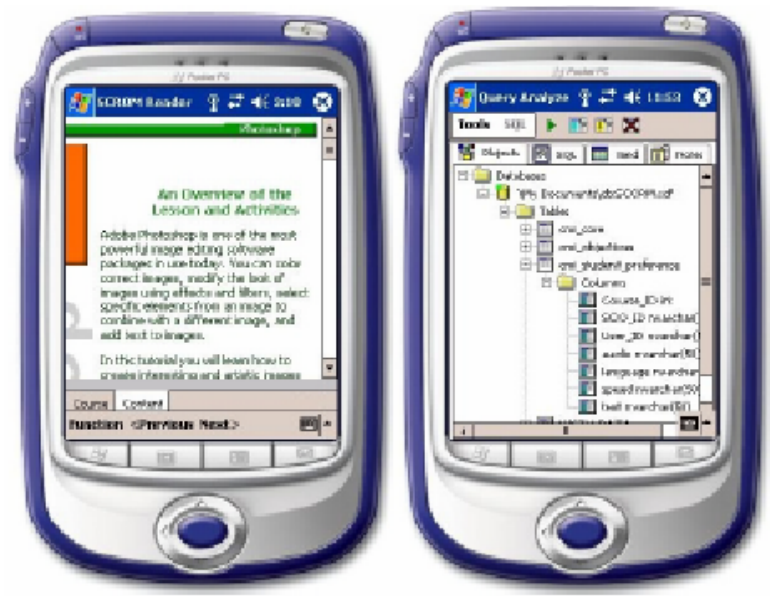

Fig. 3. SCORM PDA Reader and SCORM PDA Database 


\section{Pocket SCORM Course Caching Algorithm}

A SCORM course can be divided into several parts called clusters. A cluster includes a node of the learning content and all immediate children of the node. Every cluster has its own content size, and it is also a base unit to be loaded and to be replaced. A course developer can set the sequencing rules for each cluster, and those rules only affect the learning order of the cluster.

\subsection{Cluster Download Order}

Account to the setting of sequencing rules, it is available to forecast that what clusters the learner may read next. Loading clusters by a better order, it can increase the hit ratio of caching. With the increasing of hit ratio, a leaner do not need to download new clusters so frequently. In order to find the learning order, some of the factors are shown in the following.

Control Mode: The setting of sequencing rule. Because the setting will affect the learner's learning order, it is necessary to check the value of rules. If the "flow" flag is true, learner will be forced to read the first child node in a cluster. If the "choice" flag is true, learner can choice any child node in a cluster.

Size: The content size of each cluster. With a fix size of storage, it can contain more clusters by loading small clusters first. As the number of clusters increase the hit ratio raised, and the learner accesses the downloaded cluster more properly.

Summing up these factors can forecast the order with the following steps.

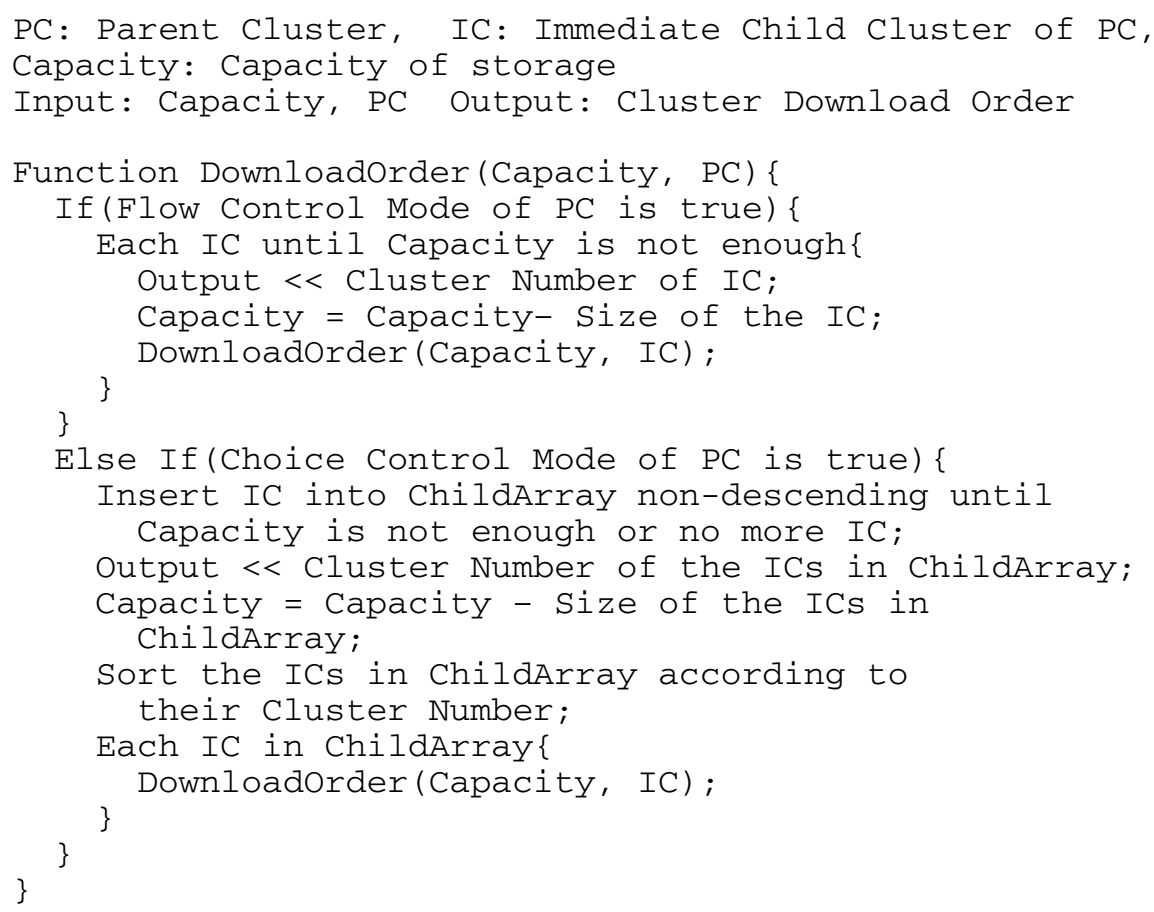




\subsection{Cluster Replacement Algorithm}

When using a storage which is smaller than the whole course, it is inevitable to replace some clusters with the new loaded ones. In order to compute what clusters should be dropped first, we assume that there has a factor called "Distance" which can determine what cluster is useless by some parameters like cluster size, download time, and so on. With the factor, it can describe the replacement as replacement algorithm.

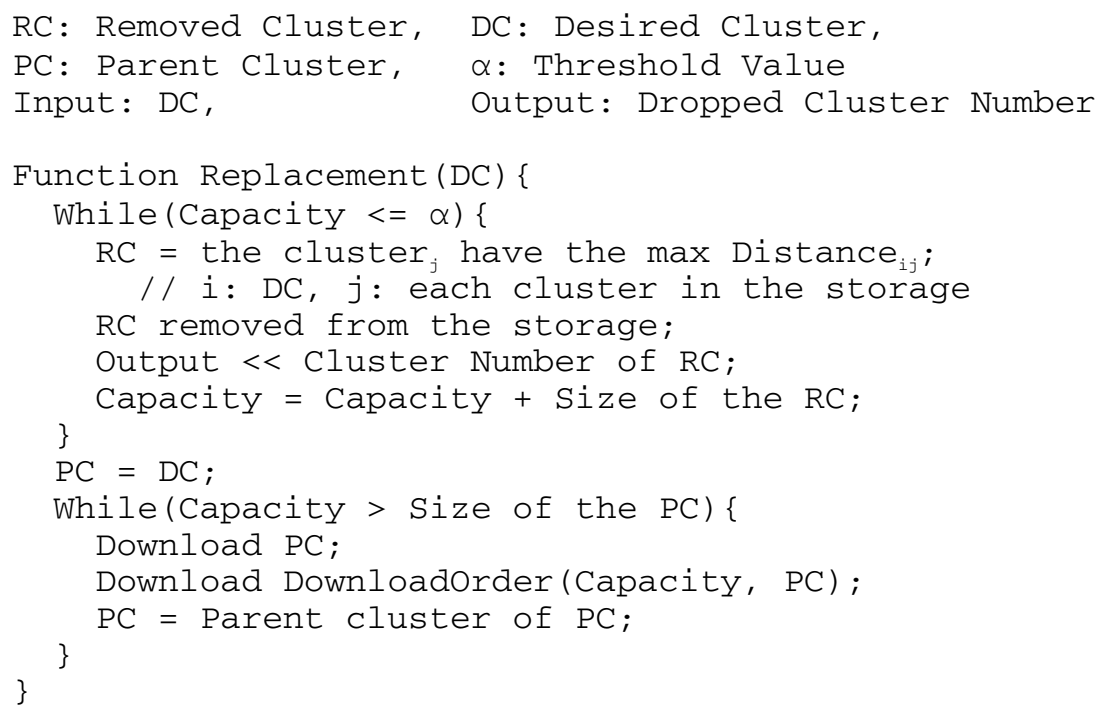

Downloading a course in good order can make the reading more smoothly and needs less replacement. With a faultless replacement algorithm, the new downloading clusters just replace the little significant ones. The two functions of the caching algorithm working together ensure that a learner does not need to reconnect to network and re-download some clusters again and again.

\section{Conclusion and Future Works}

Distance Education provides a convenient and flexible learning environment. Various kinds of distance learning methods have broken the time and space limitation. It is possible for people to learn anytime anywhere. To extend this flexibility and to make E-Learners able to learn at any location, our proposed Pocket SCORM architecture in this paper makes the dream come true. The Implemented Pocket SCORM RTE components were also introduced. We hope our proposed architecture can make ELearners to learn easier by using a pocket device which can be carried to anywhere and enable E-Learners to learn anytime. 


\section{References}

[1] Advanced Distributed Learning (ADL) (2003), http://www.adlnet.org.

[2] Oliver bohl, Dr. Jorg Schellhase, Ruth Sengler, and Prof. Dr. Udo Winand (2002), "The Sharable Content Object Reference Model (SCORM) - A Critical Review”, Proceedings of the International Conference on Computers in Education (ICCE'02)

[3] Jin-Tan David Yang, and Chun-Yen Tsai (2003), "An Implementation of SCORMcompliant Learning Content Management System - Content Repository Management System", Proceedings of the The 3rd IEEE International Conference on Advanced Learning Technologies (ICALT'03)

[4] Timothy K. Shih, Wen-Chih Chang, Nigel H. Lin, Louis H. Lin, Hun-Hui Hsu, and Ching-Tang Hsieh (2003), "Using SOAP and .NET Web Service to Build SCORM RTE and LMS", Proceedings of the17th International Conference on Advanced Information Networking and Applications (AINA'03)

[5] Peiya Liu, Liang H. Hsu, and Amit Chakraborty (2002), "Towards Automating the Generation of SCORM-Based Multimedia Product Training Manuals", Proceedings of 2002 IEEE International Conference Multimedia and Expo, 2002. (ICME '02)

[6] J. Waycott, and A. Kukulska-Hulme (2003), "Students' experiences with PDAs for reading course materials" Personal and Ubiquitous Computing Volume 7, Issue 1 (May 2003), ISSN:1617-4909

[7] Catherine Weissenborn, and Frank J. Sanchez (2001), "TekPAC (Technical Eletronic Knowledge Personal Assistant Capsule)", 2001 IEEE International Semiconductor Manufacturing Symposium

[8] Kay Sommers, Jane Hesler, and Jim Bostick (2001), "Little Guys Make a Big Splash: PDA Projects at Virginia Commonwealth University", Proceedings of the 29th annual ACM SIGUCCS conference on User services (SIGUCCS' 01)

[9] World Wide Web Consortium (W3C) (2003), http://www.w3.org/TR/soap12-part0

[10] World Wide Web Consortium (W3C) (2003), http://www.w3.org/TR/ws-arch 